\section{LA CRISIS HIPOTECARIA: IMPACTOS Y RESPUESTAS SOCIALES EN CATALUÑA}

\author{
Bálint Ábel Bereményi \\ Universitat Autònoma de Barcelona \\ ORCID iD: https://orcid.org/0000-0002-2293-3923 \\ abel.beremenyi@uab.cat \\ Irene Sabaté Muriel \\ Universitat de Barcelona \\ ORCID iD: https://orcid.org/0000-0001-8470-0765 \\ sabate.irene@gmail.com
}

\section{MORTGAGE CRISIS: SOCIAL IMPACTS AND RESPONSES IN CATALONIA}

Cómo citar este artículo/Citation: Bereményi, B. A. y Sabaté Muriel, I. (2019). La crisis hipotecaria: impactos y respuestas sociales en Cataluña. Arbor, 195 (793): a513. https://doi. org/10.3989/arbor.2019.793n3001

Recibido: 12 febrero 2016. Aceptado: 31 marzo 2016.
RESUMEN: Desde el estallido en 2008 de la burbuja inmobiliaria y financiera, el sobreendeudamiento hipotecario representa una preocupación para muchas familias españolas y catalanas, en un contexto de «nueva pobreza» caracterizada por el desempleo masivo y por unas políticas de austeridad que han conducido a muchas personas a la exclusión social. La pérdida de la vivienda tiene impacto sobre las relaciones sociales y condiciona tanto las estrategias socioeconómicas como las interpretaciones culturales del endeudamiento, en términos de estigma y de negación de una segunda oportunidad vital. Las realidades analizadas en el contexto de esta emergencia habitacional están marcadas por la amenaza del sinhogarismo, pero también por la oportunidad de la participación en un movimiento colectivo. Desde una aproximación etnográfica, los textos exploran, por una parte, la condición del endeudamiento y su impacto en las personas, y, por otra, las funciones ideológicas, políticas y socio-afectivas de un movimiento social en desarrollo.

PALABRAS CLAVE: Ejecuciones hipotecarias; sobreendeudamiento; desahucios; vivienda; crisis económica; movimientos sociales; capital social; empoderamiento; cotidianeidad; desobediencia civil; financiarización; método etnográfico.
Copyright: (C) 2019 CSIC. Este es un artículo de acceso abierto distribuido bajo los términos de la licencia de uso y distribución Creative Commons Reconocimiento 4.0 Internacional (CC BY 4.0).

ABSTRACT: Since 2008, when the housing and financial bubble gave way to the current economic crisis, mortgage overindebtedness has been a major concern for many Spanish and Catalan households. Arising in a context of "new poverty», with mass unemployment and austerity policies, this resulted in largescale social exclusion. Loss of one's home impacts people's social relations and constrains their economic strategies. There is also the cultural interpretation of indebtedness, or the related stigma, and the denial of a second chance. The realities analyzed in this special issue, within the context of this housing emergency, include the threat of homelessness, but also offer debtors an opportunity for participation in a collective movement. With an ethnographic approach, the articles explore, on the one hand, the indebted condition and its impacts on people, and, on the other, the ideological, political and socio-affective functions of an emerging social movement.

KEYWORDS: Home repossessions; (over)indebtedness; evictions; housing; economic crisis; social movements; social capital; empowerment; everyday life; civil disobedience; financialization; ethnographic method. 
En julio de 2015, el Primer Congreso Internacional de la Asociación de Antropólogos Iberoamericanos en Red (AIBR) acogió en Madrid un doble panel dedicado a la crisis hipotecaria en el Estado español, dando pie a lo que constituyó un primer foro de debate científico sobre los impactos de la crisis hipotecaria y las respuestas sociales ante ella. En el marco del panel se hicieron aportaciones que abordaron el objeto de estudio desde distintos enfoques conceptuales y teóricos pero con el denominador común de una aproximación empírica basada fundamentalmente en el método etnográfico.

El tema de los desahucios y el impacto sobre la población afectada se ha abarcado hasta el momento desde dos perspectivas de análisis en España. Por un lado se ha tratado como parte de los estudios más genéricos sobre pobreza, sinhogarismo y exclusión social y como el análisis de las políticas públicas y de las estructuras que se proponen responder a las dificultades que se derivan de esa situación. Por otra parte se ha desarrollado una línea de investigación sobre los movimientos sociales que defienden el derecho constitucional a la vivienda digna y asequible y su vulneración por parte de las instituciones financieras.

Como complemento a estas dos perspectivas, este monográfico de Arbor pretende abrir un debate sobre el fenómeno complejo y multifacético de los movimientos anti-desahucios desde las ciencias sociales de corte cualitativo. El objetivo perseguido es dar visibilidad a las experiencias e interpretaciones de los propios individuos que han participado en los movimientos, así como a los rituales y símbolos manejados desde la movilización colectiva.

A continuación pasamos a describir el contexto socioeconómico en el que se ubica el fenómeno de las ejecuciones hipotecarias y las aproximaciones que se han propuesto para analizarlo desde las ciencias sociales. Finalmente detallaremos en qué consiste la aportación específica de este monográfico.

\section{CONTEXTO: CRISIS, EXCLUSIÓN SOCIAL, SINHOGA- RISMO Y POLÍTICAS PÚBLICAS}

El largo periodo de desarrollo socioeconómico neoliberal que precede a la actual crisis se caracterizó por el aumento del consumo, así como por la reducción general de los salarios reales bajos y medios y por el endeudamiento masivo en amplias capas de la población (Lorenzo Gilsanz, 2014). Sin embargo, las crisis previas ya habían revelado la debilidad de este modelo, en el que la precariedad de la actividad productiva se relaciona con la pérdida de una inserción relacional sólida (Castel, 1997). Si bien el boom económico abrió oportunidades sin precedentes y la posibilidad percibida de una movilidad social ascendente, la debilidad del sistema de protección social ya en esas épocas se mostró insuficiente para combatir sus imperfecciones que se manifestaron en diversas formas de exclusión social. Los datos del VI Informe de la Fundación Foessa (Laparra Navarro y Pérez Eransus, 2008) afirman que las prestaciones sociales durante los años de la bonanza económica fueron eficaces en la reducción de la pobreza severa (87\%), pero en cambio solo redujeron un $47 \%$ la pobreza moderada. Entre 1997 y 2006, a pesar del crecimiento sin precedentes del PIB (>3\%), el poder adquisitivo medio prácticamente no creció y se generó más desigualdad entre los segmentos más ricos y más pobres de la población española (Lorenzo Gilsanz, 2014, p. 44). El desempleo no ha afectado a todos los sectores sociales de la misma manera (Laparra Navarro y Pérez Eransus, 2012, p. 20). En la construcción y los servicios relacionados el impacto ha sido extremadamente grande, afectando principalmente a los hombres, a los más jóvenes y a aquellos que menos estudios tenían. En el sector servicios el desempleo de las mujeres ha llegado a niveles superiores a los de los hombres (Maier, 2011, citado en Laparra Navarro y Pérez Eransus, 2012). El paro entre los jóvenes menores de 25 años alcanzó niveles nunca vistos antes (en 2013, primer trimestre (T1), superó el 56\% según la Encuesta de Población Activa (http://www.ine.es/jaxiT3/Tabla.htm?t=4247)). Por otra parte, las familias inmigrantes y miembros de minorías, principalmente la etnia gitana (Laparra Navarro, 2008), que en años anteriores se incorporaban al mercado laboral en una proporción creciente, se vieron como los principales perdedores de la crisis debido a sus peores condiciones contractuales y a los sectores donde encontraron empleo (véase Pajares, Inmigración y mercado de trabajo. Informe 2010).

El "tardío" estado del bienestar español (Moreno, 2007), o "régimen mediterráneo de protección social" (Moreno y Serrano, 2009), fundamenta el acceso a la protección de rentas principalmente en la participación o no en el mercado laboral (actual y pasada). Consecuentemente, la pérdida del empleo o la inclusión en la economía informal puede conducir a la gradual exclusión de la "última red de protección social" (Arriba González de Durana y Pérez Eransus, 2007). Este régimen considera a la familia como un factor esencial de "microsolidaridad", y en este sentido su presencia complementa algunas funciones de provisión del Estado. Sin embargo, en un número creciente de hogares el desempleo de varios o de todos los miembros activos ha im- 
pedido a la familia cumplir esa función protectora. Entre 2005 y 2012 la proporción de hogares donde todos sus miembros activos se encontraban desempleados pasó del 3,1\% al 9,9\% (Carbonero Muñoz y Ruíz Vega, 2016, p. 42). Al mantenerse con los años los altos niveles de desempleo, las prestaciones asistenciales han ido sustituyendo a las prestaciones de carácter contributivo. Puesto que el crecimiento económico de la etapa anterior no se había aprovechado para consolidar un modelo más coherente de protección social, la precariedad se ha consolidado como un "rasgo de la estructura social" a raíz la crisis (Lorenzo Gilsanz, 2014, p. 44).

\section{PERSPECTIVAS PARA EL ANÁLISIS DE LA CRISIS HI- POTECARIA Y LA EMERGENCIA HABITACIONAL EN EL ESTADO ESPAÑOL}

Desde el estallido de la burbuja inmobiliaria y financiera el sobreendeudamiento hipotecario representa una preocupación para muchos grupos domésticos en tiempos de desempleo masivo y de austeridad presupuestaria (Laparra Navarro y Pérez Eransus, 2012). Las condiciones de habitabilidad han sufrido un empeoramiento para amplias capas de la población, especialmente para las familias más vulnerables (Laparra Navarro y Pérez Eransus, 2012), para las cuales los años anteriores de bonanza económica ofrecían la posibilidad de obtener un inmueble de propiedad asumiendo la carga de una hipoteca. Sastre y Fernández-Sánchez (2011) ofrecen datos sobre la reducción significativa del gasto en los hogares en estos años de crisis, lo que en muchos casos dificulta cubrir las necesidades básicas de la familia, e implica a menudo el deterioro de la calidad de la vivienda como una forma de privación relevante. La reducción de gastos ha obligado a muchas familias a acumular impagos de facturas, sumiéndoles en una espiral que para muchos ha desembocado en la pérdida de la vivienda. El mayor aumento de la morosidad entre 2007 y 2010 se observó en relación con la hipoteca y el alquiler, afectando a un 4,5\% de los hogares en el Estado español (Laparra Navarro y Pérez Eransus, 2012, p. 113). Asimismo, en el periodo 2007-2013 se multiplicó el número de hogares que, una vez sufragados los gastos de vivienda, se situaban en condiciones de pobreza severa (Laparra Navarro, 2014, p. 161). Desde una perspectiva estructural, la pérdida masiva del hogar familiar no es más que un indicador de la "nueva pobreza" emergente (Paugam y Hernández Díaz, 2007) en un contexto de inestabilidad laboral, recesión económica y desempleo estructural que conduce a formas diversas de exclusión social. De hecho, perder la propiedad del hogar familiar o estar en riesgo de hacerlo representa una de las muy diversas situaciones de exclusión residencial (Cabrera Cabrera, 2009, p. 188). La tercera categoría de la tipología ETHOS (European Typology on Homelessness) se refiere a la "vivienda insegura" como una de las formas de sinhogarismo, consistente en "vivir en una vivienda sin título legal (vivir temporalmente con familiares o amigos de forma involuntaria, vivir en una vivienda sin contrato de arrendamiento)" y haber recibido "notificación legal de abandono de la vivienda". También se mencionan en esta categoría las "personas viviendo bajo amenaza de desahucio" (Cabrera y Cabrera, 2009; Cabrera, Rubio y Blasco, 2008, p. 18).

En efecto, entre 2007 y el tercer trimestre de 2015 se iniciaron en España 656.839 procesos de ejecución hipotecaria, según el Consejo General del Poder Judicial (Figura 1).

Desde la perspectiva de los propietarios la amenaza de la pérdida de la vivienda habitual, con la consiguiente negación de la satisfacción de una necesidad básica como es el cobijo, se suma a la experiencia de los deudores de ser culpados de su propia situación y estigmatizados por su fracaso vital, tanto por su entorno próximo como en las narrativas públicas establecidas. Un fracaso que supone el abandono de unos proyectos vitales y de unas expectativas de movilidad social ascendente que hoy se revelan inviables (Bereményi y Carrasco, 2018). La realidad de la "generación de las ejecuciones hipotecarias" (Bowdler, Quercia y Smith, 2010), ampliamente discutida en los Estados Unidos, se enmarca de unas condiciones estructurales peculiares en el Estado español y concretamente en Cataluña, debido al alto nivel de movilidad social ascendente anterior a la crisis y a la subsecuente movilidad descendente, sobre todo económica, de una parte significativa de las clases medias y bajas (Peláez-Paz, 2014; Requena y Stanek, 2015). La pérdida de la tenencia de la vivienda es una prueba fehaciente de esa movilidad descendente. Las consecuencias del fracaso personal y colectivo de amplias capas sociales se agrava todavía más por las peculiaridades de la legislación hipotecaria española, que niega a los deudores una segunda oportunidad vital al relegarles a la condición de morosos de forma indefinida (Colau y Alemany, 2012), y por la ineficacia de las políticas de protección social, que han tardado en responder a la emergencia habitacional y al desencadenamiento de procesos de exclusión social (Arriba González de Durana y Pérez Eransus, 2007). Junto a los avatares del procedimiento de ejecución del inmueble que es garantía del préstamo hipotecario, el proceso de pérdida de la vivienda provoca cambios fundamentales tanto en las estrategias socioeconómi- 
Figura 1. Evolución de las ejecuciones hipotecarias presentadas por el Tribunal Superior de Justicia (TSJ). Período 2007-2015

\section{Ejecuciones hipotecarias presentadas por TSJ 2007-2015}

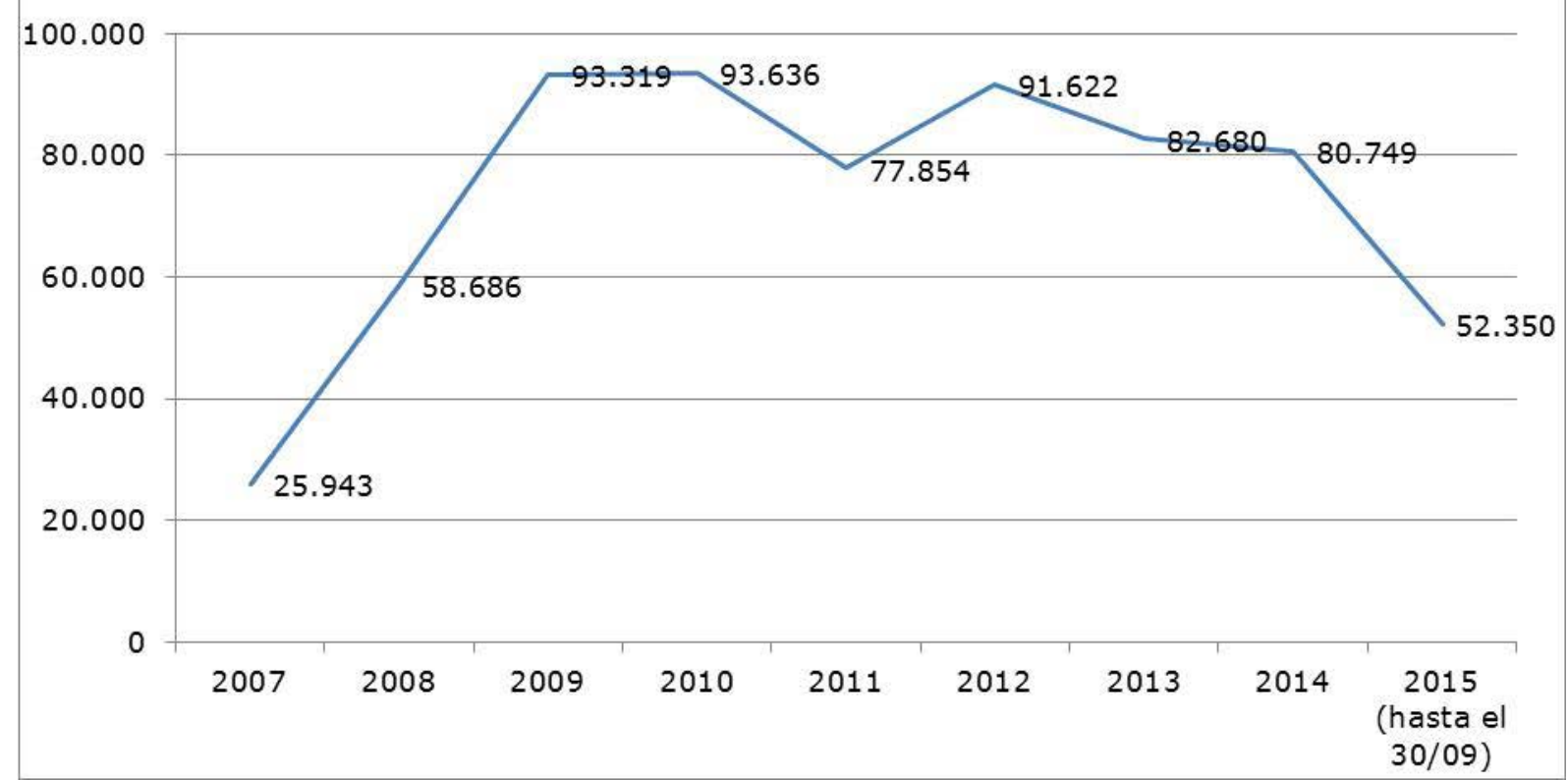

Fuente: Consejo General del Poder Judicial. Sección de Estadística Judicial. Datos sobre el efecto de la crisis en los órganos judiciales (http:// www.poderjudicial.es/cgpj/es/Temas/Estadistica-Judicial/Informes-estadisticos-periodicos/Datos-sobre-el-efecto-de-la-crisis-en-los-organos-judiciales---Datos-desde-2007-hasta-tercer-trimestre-de-2015).

cas de las personas y de los grupos domésticos como en sus relaciones sociales y en sus oportunidades de reproducción social, sobre las que se produce una presión que amenaza con dislocarlas. En tal contexto se crean y recrean interpretaciones y significados culturales en el intento de dar sentido a una cotidianeidad marcada por el estrés y la incertidumbre (Castel, 1997). En muchos casos la pérdida de la vivienda, o la amenaza de esta, repercute negativamente en la salud mental y física de los afectados, así como en sus oportunidades presentes y futuras de bienestar e inserción social (Laparra Navarro, 2014). Basta mencionar tan solo un dato del Informe del observatorio DESC publicado en 2015 (Emergència habitacional a Catalunya. Impacte de la crisi hipotecària en el dret a la salut i els drets dels infants), que señala cómo detrás de cada diez ejecuciones hipotecarias hay nueve menores (p. 22). El Informe sobre España 2016 de la Comisión Europea menciona las consecuencias sociales de las ejecuciones hipotecarias como uno de los efectos más importantes de la crisis (Baptista, 2016) y expresa su crítica a la ejecución de la Estrategia Nacional Integral para Personas sin Hogar 2015-2020 y su evaluación.
Por otra parte, la emergencia de movimientos sociales en respuesta a la proliferación de las ejecuciones hipotecarias, entre los que destaca el caso de la Plataforma de Afectados por la Hipoteca (PAH), vehicula la búsqueda de salidas para el sobreendeudamiento hipotecario. Los movimientos exigen soluciones a la clase política y a las élites bancarias, que son identificadas como responsables de la crisis por parte de amplios sectores de la sociedad española.

Diversos investigadores se plantean en qué sentido la $\mathrm{PAH}$ y otros movimientos sociales en el ámbito de la vivienda han cambiado la experiencia de ciudadanía de las personas. La concepción de 'ciudadanía' acuñada por Lefèbvre (1968) y desarrollada por diferentes autores se formula en tres niveles. Primero, la ciudadanía como modelo social ideal; segundo, como una reclamación al sistema del ejercicio de derechos ciudadanos; y por último, como la participación activa de una masa crítica para cambiar el sistema. Para algunos (Swyngedouw, 2005; Swyngedouw, 2011, citado en Rogers y Bailey, 2013, p. 5), el verdadero derecho a la ciudad (Lefèbvre, 1968) corresponde a esta tercera forma de ciudadanía activa y performativa. Rogers y Bai- 
ley (2013) destacan la importancia de identificar qué actores (individuos o grupos), en qué espacios y de qué manera son autorizados a actuar, y hasta qué punto se reconoce su actuación como legítima. En esta misma línea, autores como Mouffe (2005) o Rancière (Rancière y Panagia, 2000) no ubican la ciudadanía en los espacios de la política institucional democrática sino que destacan cómo la ciudadanía, lejos de ser normativa, incluye derechos, responsabilidades y acciones cívicas de escalas variadas. Esta definición contrasta con la idea neoliberal del ciudadano como consumidor responsable. Los "consumidores deficitarios" (flawed citizens), como los presenta Bauman (1998), son aquellos, típicamente la población de rentas bajas, que no son capaces de cumplir con sus responsabilidades económicas, y como tales ven cuestionada su responsabilidad. En el contexto de la crisis financiera es justamente este último modelo de ciudadanía, la del derecho a elegir mediante el consumo (Jubas, 2009), la que se niega a aquellas familias que primero pierden su trabajo y luego su capacidad de cumplir con sus deberes como ciudadanos. Su culpabilización, humillación y expulsión se hace palpable en el proceso de ejecución hipotecaria, en que los servicios públicos no protegen, o no suficientemente, la integridad de este "ciudadano". La PAH, en este sentido, ha revelado su capacidad para ofrecer, aparte de soluciones prácticas vitales, un modelo alternativo de ciudadanía a través de la participación activa de una masa crítica con el objetivo común de repensar y reivindicar los fundamentos mismos de una nueva ciudadanía.

La crisis hipotecaria y sus consecuencias, por su visibilidad y mediatización, han generado mucho interés entre los científicos sociales. En los últimos años se han multiplicado los trabajos académicos, las comunicaciones en reuniones científicas y los proyectos de investigación que abordan este objeto de estudio, ya sea de forma monográfica o situándolo en el contexto más general de la exclusión y la fractura social (Laparra Navarro y Pérez Eransus, 2012; Lorenzo Gilsanz, 2014) y de su expresión más extrema, el sinhogarismo (Cabrera Cabrera, 2009; Carbonero Muñoz y Ruiz Vega, 2016; Sales i Campos, Diagnosis 2013. Las personas sin hogar en la ciudad de Barcelona y la evolución de los recursos de la Red de Atención a Personas sin Hogar), en el marco de la también creciente bibliografía sobre la crisis económica y las políticas de austeridad en el sur de Europa, sobre sus repercusiones sociales y sobre las reacciones ante ella (Albertos y Sánchez Hernández, 2014; Knight y Stewart, 2016; Narotzky, 2016).

En especial las particularidades de la PAH como un caso conspicuo de nuevo movimiento social han sus- citado un enorme interés tanto dentro como fuera de las fronteras del Estado (Cano y Etxezarreta, 2014; García Lamarca, 2017; Mangot, 2013; Mir García, França, Macías y Veciana, 2013; Parcerisa, 2014; Sabaté, 2014; Sabaté, 2016; Suárez, 2014). La bibliografía específicamente generada en torno a este movimiento ha contribuido en buena medida a desafiar los relatos promovidos por el discurso público dominante y al mismo tiempo ha conseguido sentar un canon para las aproximaciones alternativas a la problemática hipotecaria (Colau y Alemany, 2012; Macías, 2013; véanse también los informes Emergència habitacional a Catalunya. Impacte de la crisi hipotecària en el dret a la salut i els drets dels infants de 2015, Emergencia habitacional en el Estado español. La crisis de las ejecuciones hipotecarias y los desalojos desde una perspectiva de derechos humanos de 2013 y Colau y Alemany (2013), Retrospectiva sobre desahucios y ejecuciones hipotecarias en España, estadísticas oficiales e indicadores).

Sin embargo, todavía no podemos hablar de un cuerpo coherente de aportaciones analíticas sobre la morosidad, las ejecuciones hipotecarias y sus efectos socioculturales en el Estado español. Por ello, el objetivo de este monográfico es abrir un debate en torno a los marcos interpretativos y de referencia que permitan entender mejor las experiencias y respuestas de la población afectada y del resto de actores sociales implicados. En última instancia, se trata tanto de visibilizar y analizar los conflictos y complejidades del fenómeno social como de identificar perfiles y tendencias que permitan abordar la problemática a través de cambios legislativos y mediante el diseño de políticas.

\section{CONTRIBUCIONES DE ESTE NÚMERO MONOGRÁFICO}

Los textos publicados en este monográfico proponen una aproximación etnográfica a la realidad social observada. Los autores, todos ellos con formación antropológica o en disciplinas afines, pretenden profundizar en el conocimiento sobre las personas integrantes de la $\mathrm{PAH}$ y sobre la dimensión colectiva de este movimiento centrándose en las interpretaciones emic de sus protagonistas. En consonancia con esto, los textos se basan principalmente en datos recogidos durante un dilatado trabajo de campo etnográfico (Hammersley y Atkinson, 1994), asistiendo a asambleas y acciones colectivas de diferente carácter, en algunos casos asumiendo activamente responsabilidades en el grupo observado. Las técnicas de recogida de datos más generalmente aplicadas por los autores han sido la observación participante (Bernard, 2011, p. 342; Guber, 2001), las entrevistas 
individuales y grupales (Guber, 2001), así como la exploración documental (Valles, 1999). Los autores toman partido por una aproximación empírica a la realidad social que privilegia las experiencias directas y las trayectorias de los actores sociales. Para ello aplican técnicas interpretativas de análisis, adoptando una perspectiva crítica.

Asimismo, conviene explicitar la pertinencia de la ubicación en Cataluña de los estudios etnográficos aquí reunidos. El caso catalán nos permite percibir de manera particularmente nítida el trauma social provocado por el contraste entre un período anterior de bonanza económica -caracterizado entre otras cosas por el auge de la construcción, la burbuja de precios inmobiliarios y el acceso a la propiedad por una amplia mayoría social, incluidas las capas de población en situaciones más precarias- y la actual crisis, durante la que el desempleo y la falta de protección social han enviado a muchas familias, a menudo deudoras de una hipoteca, a la exclusión social y residencial. Es en Cataluña, además, donde tuvo su origen la primera asamblea de la $\mathrm{PAH}$, creada en Barcelona en 2009, en continuidad con movimientos anteriores que ya venían reivindicando el derecho a la vivienda en la coyuntura económica anterior (Colau y Alemany, 2012). La experiencia de Barcelona y de otras poblaciones del área metropolitana resulta así especialmente significativa a la hora de palpar la inspiración, objetivos y logros de este movimiento social. Como es sabido, el modelo de la PAH de Barcelona ha sido replicado, con las pertinentes adaptaciones, en el resto de la geografía española, hasta alcanzar las casi dos centenares de asambleas en todo el Estado.

En lo referente al marco conceptual, la colección de textos aquí presentados se inspira en referentes y debates procedentes de la sociología, la antropología, la geografía, la politología y otras disciplinas afines. El fenómeno de las ejecuciones hipotecarias se estudia, así, a la luz de distintos conceptos y teorías. Entre ellos encontramos las nociones de capital social y movilidad social, el empoderamiento, los movimientos sociales, los regímenes de movilidad y los procesos migratorios, así como las discusiones acerca de las tecnologías del poder y la financiarización de la vida cotidiana y de las economías domésticas bajo el capitalismo contemporáneo.

En relación con esto último, diversos autores (Aalbers, 2008) han señalado el acceso a la vivienda de propiedad y su consiguiente participación en los mercados inmobiliarios e hipotecarios como uno de los principales vectores de la financiarización, entendida como la creciente penetración, a lo largo de las últimas décadas, de las dinámicas financieras en ámbitos como el de las economías domésticas y en sectores sociales como las clases populares, que hasta hace poco se sustraían a esta influencia. El texto de Melissa García Lamarca argumenta, en este sentido, que la financiarización de la vivienda no puede entenderse sin analizar también la financiarización de la vida misma, es decir, de las subjetividades y los cuerpos de quienes se ven implicados en el proceso. El fenómeno de la financiarización debe entenderse según esto como una tecnología del poder (Foucault, 2008) que tiene la capacidad de "hipotecar las vidas", es decir, de disciplinar y proletarizar a los sujetos que aspiran a convertirse en propietarios de una vivienda, convirtiéndose en víctimas de una forma secundaria de explotación (Harvey, 1982). Ese proceso de disciplina y proletarización es el que explica las circunstancias de las que, tras el fin del boom económico, parten los deudores que se ven incapaces de cumplir con las cuotas hipotecarias.

El texto de Georgios Azis examina cómo, a partir del impago, o ante su perspectiva cercana, se hace evidente de pronto una circunstancia que tendía a pasar desapercibida durante los tiempos de bonanza: lo que el autor propone denominar la subordinación financiera, entendida como la exposición directa de la cotidianeidad, es decir, de las necesidades y aspiraciones reproductivas de las personas y los grupos domésticos, a los mecanismos de la expropiación y de la acumulación financiera (Lapavitsas, 2009). La violencia estructural implicada en esta relación asimétrica de deuda y crédito (Graeber, 2011) deviene súbitamente visible al desencadenarse el proceso de ejecución hipotecaria, un proceso que puede completarse con la violencia abierta del desahucio. Los deudores, en algunos casos, buscan la intervención en su relación con la entidad bancaria de un movimiento social como la PAH, que cumple una función de defensa y reclamación de derechos mediante la interposición entre las partes implicadas en la relación crediticia. Esta interposición, no obstante, no estará libre de tensiones y conflictos, en buena medida por implicar una reestructuración de la cotidianeidad de los deudores que no en todos los casos será posible.

El texto de Irene Sabaté retoma las nociones antropológicas de la deuda y el crédito (Graeber, 2011; Gregory, 2012; Guyer, 2012; Mauss, 1923/1979) para analizar en qué medida el aumento de la morosidad hipotecaria y la proliferación de las ejecuciones puede interpretarse como una transgresión de la obligación de devolver a escala masiva. La autora analiza las implicaciones de tal transgresión, por un lado, como el resultado de unos constreñimientos estructurales que incluyen la disminución drástica de los ingresos y la falta de una legisla- 
ción y unas políticas que amparen a los deudores en las nuevas circunstancias. Por otro lado, la transgresión de la obligación de devolver va acompañada de una resignificación de la deuda como ilegítima, ya sea debido a las condiciones en que se contrajo, ya sea por la asfixia que el sobreendeudamiento está produciendo en la actualidad, en el sentido de impedir la satisfacción de otras necesidades urgentes.

La articulación entre legalidad y legitimidad se analiza también en el texto de Marina Pera, quien, sobre la base de una investigación empírica entre los participantes en ocupaciones colectivas de edificios propiedad de las entidades financieras por parte de personas ya desahuciadas, reflexiona sobre la valoración que los protagonistas hacen de su propio acto de apropiación ilegal de un inmueble. En su mayoría personas sin experiencia activista previa, no identificadas con los idearios y reivindicaciones políticas del movimiento okupa, los entrevistados justifican su comportamiento, entendido como un acto de desobediencia civil (Rawls, 1971), sobre la base de la necesidad perentoria de obtener un cobijo y confían en la posibilidad de poder regularizar su situación mediante el pago de un alquiler que resulte proporcional a su situación económica.

La participación de ocupaciones colectivas, y el consiguiente acceso a una vivienda alternativa tras la ejecución de la propia, es tan solo uno de los recursos puestos al alcance de los participantes en la PAH. En su artículo, Bálint Ábel Bereményi y Elena Montero se interrogan de manera más general acerca de la naturaleza del capital social y de las oportunidades de movilidad social proporcionadas por la participación en el movimiento. El encuentro con otras personas en la misma situación permite el acceso a nuevas redes de apoyo y solidaridad que permiten hacer frente a una situación de emergencia socioeconómica y afectiva. Sin embargo, según los autores, la función de "ventaja" del capital social (de Souza Briggs, 1998) solo se activará en los casos en que se produzca un auténtico empoderamiento, en el sentido de una toma de conciencia del carácter sistémico de la problemática y de fortalecimiento de la identidad colectiva de los "afectados" como sujeto político.

Finalmente, Maka Suárez se interesa por la metodología de la PAH a la hora de comunicar su discurso a la opinión pública, a los medios de comunicación y a las élites políticas y bancarias. Concretamente la autora se centra en el uso de lo que denomina objetos desobedientes (Flood y Grindon, 2014), en referencia a los elementos materiales utilizados durante movilizaciones y acciones públicas de carácter performativo. El uso de tales objetos, entre los que destacan los grandes círculos de cartón rojos y verdes utilizados durante la campaña de presión a los parlamentarios para que apoyaran la Iniciativa Legislativa Popular impulsada por la PAH entre otras entidades en 2013 , es un aspecto clave de la puesta en escena de un descontento popular que se concreta en la reivindicación del derecho a la vivienda y en un cuestionamiento de las bases sociales de la moralidad de la deuda (Graeber, 2011).

La diversidad de las inquietudes y aproximaciones aquí reunidas es reflejo de una efervescencia investigadora suscitada por una problemática especialmente grave y urgente que, si bien ha emergido también en otros puntos del globo, como los Estados Unidos (Agnew, 2010; Bowdler et al., 2010; Crowley, 2003; Martin y Niedt, 2015; Schuetz, Been y Ellen, 2008; Stout, 2015, 30 de marzo) u otros estados europeos como Irlanda (Waldron y Redmon, 2014), presenta en el caso español suficientes peculiaridades como para ser considerada de forma específica. Gran parte de estas particularidades derivan, por un lado, de los modelos político, urbanístico y productivo aplicados en España desde la transición a la democracia o incluso desde antes (Coq-Huelva, 2013; López y Rodríguez, 2010; Naredo, 2009). Por otro lado, la centralidad que la vivienda de propiedad ha adquirido para las economías domésticas y los proyectos vitales de los ciudadanos en la historia reciente (Allen, Barlow, Leal, Maloutas y Padovani, 2004; Palomera, 2013) explican también la enorme repercusión social de las ejecuciones hipotecarias. A ello se añade, sin duda, la enorme expectación generada en el ámbito internacional en torno a un movimiento social, la Plataforma de Afectados por la Hipoteca, que, desde su creación en Barcelona en el año 2009 , no ha dejado de exigir y proponer soluciones para el sobreendeudamiento y la crisis hipotecaria, entendidos como una grave amenaza para la reproducción social de un amplio sector social cuyas oportunidades de futuro se encuentran en grave peligro.

\section{AGRADECIMIENTOS}

La recopilación de este monográfico se ha realizado en el marco del proyecto I+D+i MOVIBAR (Ministerio de Educación, Cultura y Deporte, CSO 2012-34285), cuya investigadora Principal es la doctora Sílvia Carrasco de la Universitat Autònoma de Barcelona; con una beca PostPhD Grant 2014 de la Wenner-Gren Foundation y con financiación de la Generalitat de Catalunya (ayudas para Grupos de Investigación Consolidados - GRC 2014) concedida al Grup d'Estudis sobre Reciprocitat de la Universitat de Barcelona, así como al Centre d’Estudis i Recerca en Migracions de la Universitat Autònoma de Barcelona). 


\section{BIBLIOGRAFÍA}

Aalbers, M. B. (2008). The financialization of home and the mortgage market crisis. Competition and Change, 12 (2), pp. 148-166.

Agnew, S. (2010). The Impact of Affordable Housing on Communities and Households. Minnesota Housing Finance Agency Research and Evaluation Unit. Disponible en https://medinamn. us/wp-content/uploads/2014/04/ The-Impact-of-Affordable-Housing-onCommunities-MHFA.pdf

Albertos, J. M. y Sánchez Hernández, J. L. (2014). Geografía de la crisis económica en España. Valencia: Publicacions de la Universitat de València.

Allen, J., Barlow, J., Leal, J., Maloutas, Th. y Padovani, L. (2004). Housing and Welfare in Southern Europe. Oxford: Blackwell Publishing. https://doi. org/10.1002/9780470757536

Arriba González de Durana, A. y Pérez Eransus, B. (2007). La última red de protección social en España: prestaciones asistenciales y su activación. Política y Sociedad, 44 (2), pp. 115-133.

Baptista, I. (2016). Strategically moving forward in combatting homelessness in Spain. European Journal of Homelessness, 10 (2), pp. 89-110.

Bauman, Z. (1998). Work, Consumerism and the New Poor. Buckingham: Open University Press.

Bereményi, B. Á. y Carrasco, S. (2018). Caught in the triangle of mobility: social, residential and pupil mobility. British Journal of Sociology of Education, 39 (1), pp. 32-46. https://doi.org/10.1080 /01425692.2017.1302316

Bernard, H R. (2011). Research methods in anthropology: Qualitative and quantitative approaches (4. a ed.). Lanham, MD: Altamira Press.

Bowdler, J., Quercia, R. y Smith, D. A. (2010). The Foreclosure Generation. The LongTerm Impact of the Housing Crisis on Latino Children and Families. Washington D.C.: NCLR - National Council of La Raza.

Cabrera Cabrera, P. J. (2009). La acción social con personas sin hogar en la España del siglo XXI. Avances y retos en la última década. En Laparra Navarro, M. y Pérez Eransus, B (coords.). La exclusión social en España. Un espacio diverso y disperso en intensa transformación. Madrid: Fundación Foessa, pp. 173-219.
Cabrera, P., Rubio, M. J. y Blasco, J. (2008). ¿Quién duerme en la calle? Una investigación social y ciudadana sobre las personas sin techo. Barcelona: Fundació Caixa Catalunya.

Cano, G. y Etxezarreta, A. (2014). La crisis de los desahucios en España. Respuestas institucionales y ciudadanas. Revista de Economía Crítica, 17, pp. 44-57.

Carbonero Muñoz, D. y Ruíz Vega, A. (2016). Evolución en los procesos e itinerarios de las personas sin hogar en España: ¿Retorno al pasado? EMPIRIA. Revista de Metodología de Ciencias Sociales 34, pp. 39-78. https://doi.org/10.5944/empiria.34.2016.16522

Castel, R. (1997). La metamorfosis de la cuestión social. Una crónica del salariado. Barcelona: Paidós.

Colau, A. y Alemany, A. (2012). Vides hipotecades. De la bombolla immobiliària al dret a l'habitatge. Barcelona: Angle Editorial.

Coq-Huelva, D. (2013). Urbanisation and Financialisation in the Context of a Rescaling State: The Case of Spain. Antipode, 45 (5), pp. 1213-1231. https://doi. org/10.1111/anti.12011

Crowley, S. (2003). The Affordable Housing Crisis: Residential Mobility of Poor Families and School Mobility of Poor Children. The Journal of Negro Education, 72 (1), pp. 22-38. https://doi. org/10.2307/3211288

Flood, C. y Grindon, G. (2014). Introduction. En: Flood, C. y Grindon, G. (eds.). Disobedient Objects. London: V\&A Publishing, pp. 6-25.

Foucault, M. (2008). The Birth of Biopolitics: Lectures at the Collège de France, 19781979. Hampshire: Palgrave Macmillan.

García Lamarca, M. (2017). From Occupying Plazas to Recuperating Housing Insurgent Practices in Spain. International Journal of Urban and Regional Research, 41 (1), pp. 37-53. https://doi. org/10.1111/1468-2427.12386

Graeber, D. (2011). Debt. The first 5,000 years. New York: Melville House.

Gregory, C. A. (2012). On money debt and morality: some reflections on the contribution of economic anthropology. Social Anthropology, 20 (4), pp. 380396. https://doi.org/10.1111/j.1469 $8676.2012 .00225 x$
Guber, R. (2001). La etnografía. Método, campo y reflexividad. Buenos Aires: Norma.

Guyer, J. I. (2012). Obligation, binding, debt and responsibility: provocations about temporality from two new sources. Social Anthropology, 20 (4), pp. 491-501. https://doi. org/10.1111/j.1469-8676.2012.00217.x

Hammersley, M. y Atkinson, P. (1994). Etnografía: métodos de investigación. Madrid: Paidós.

Harvey, D. (1982). The Limits to Capital. London: Verso.

Jubas, K. (2009). Conceptual con/fusion in democratic societies: Understandings and limitations of consumercitizenship. Journal of Consumer Culture, 7 (2), pp. 231-254. https://doi. org/10.1177/1469540507077683

Knight, D. M. y Stewart, C. (2016). Ethnographies of Austerity: Temporality, Crisis and Affect in Southern Europe. History and Anthropology, 27 (1), pp. 1-18. https://doi.org/10.1080/02757206.201 5.1114480

Laparra Navarro, M. (2008). La comunidad gitana: el grupo étnico más excluido en la sociedad española. En Laparra Navarro, M. y Pérez Eransus, B. (coords.). La exclusión social en España. Un espacio diverso y disperso en intensa transformación. Madrid: Fundación Foessa, pp. 405-423.

Laparra Navarro, M. y Pérez Eransus, B. (coords.) (2012). Crisis y fractura social en Europa. Causas y efectos en España. Barcelona: Obra social «La Caixa».

Laparra Navarro, M. (2014). La fractura social se ensancha: intensificación de los procesos de exclusión en España durante 7 años. En Lorenzo Gilsanz, F. (coord.). VII Informe sobre exclusión y desarrollo social en España. Madrid: Fundación Foessa, pp. 152-255.

Laparra Navarro, M. y Pérez Eransus, B. (coords.) (2008). La exclusión social en España. Un espacio diverso y disperso en intensa transformación. Madrid: Fundación Foessa.

Lapavitsas, C. (2009). Financialised Capitalism: Crisis and Financial Expropriation. Historical Materialism, 17 (2), pp. 114-148. https://doi. org/10.1163/156920609X436153

Lefèbvre, H. (1968). The Right to the City. Paris: Anthropos. 
López, I. y Rodríguez, E. (2010). Fin de ciclo. Financiarización, territorio y sociedad de propietarios en la onda larga del capitalismo hispano (1959-2010). Madrid: Traficantes de Sueños.

Lorenzo Gilsanz, F. (coord.). (2014). VII Informe sobre exclusión y desarrollo social en España. Madrid: Fundación Foessa.

Macías, C. (2013). Del empobrecimiento a la autotutela de derechos. El caso de la PAH. EI Viejo Topo, 306-307, pp. 44-48.

Maier, F. (2011). Will the crisis change gender relations in labour markets and society? Journal of Contemporary European Studies, 19 (1), pp. 83-95. https://doi.org/1 $0.1080 / 14782804.2011 .554197$

Mangot, LI. (2013). La Plataforma de Afectados por la Hipoteca. De la crisis a la estafa. Del Prozac al empoderamiento. Clivatge. Estudis i testimonis sobre el conflicte i el canvi socials, 2, pp. 56-87. Disponible en http://revistes. ub.edu/index.php/clivatge/article/ view/10021/12890

Martin, I. W. y Niedt, Ch, (2015). Foreclosed America. California: Standford University Press.

Mauss, M. (1923/1979). Ensayo sobre los dones. Razón y forma del cambio en las sociedades primitivas. En: Mauss, M. Sociología y Antropología. Madrid: Tecnos, pp. 155-263.

Mir García, J., França, J., Macías, C. y Veciana, P. (2013). Fundamentos de la Plataforma de Afectados por la Hipoteca: activismo, asesoramiento colectivo y desobediencia civil no violenta. Educación Social. Revista de Intervención Socioeducativa, 55, pp. 52-61.

Moreno, L. (2007). Europa social, bienestar en España y la 'malla de seguridad'. En Espina, A. (coord.). Estado de Bienestar y competitividad. La experiencia europea. Madrid: Fundación Carolina / Siglo XXI, pp. 445-511.

Moreno, L. y Serrano, A. (2009). Modelo social europeo y políticas sociales: una evaluación formativa institucional. Gestión y análisis de políticas públicas, 2, pp. 11-32.

Mouffe, Ch. (2005). The Democratic Paradox. New York: Verso.

Naredo, J. M. (2009). La cara oculta de la crisis. El fin del boom inmobiliario y sus consecuencias. Revista de Economía Crítica, 7, pp. 313-340.
Narotzky, S. (2016). Between Inequality and Injustice: Dignity as a Motive for Mobilization During the Crisis. History and Anthropology, 27 (1), pp. 74-92. https://doi.org/10.1080/02757206.201 5.1111209

Palomera, J. (2013). How Did Finance Capital Infiltrate the World of the Urban Poor? Homeownership and Social Fragmentation in a Spanish Neighborhood. International Journal of Urban and Regional Research, 38 (1), pp. 218235. https://doi.org/10.1111/14682427.12055

Parcerisa, LI. (2014). La PAH: Un moviment social contrahegemònic? Oxímora. Revista Internacional de Ética y Política, 4, pp. 23-40.

Paugam, S. y Hernández Díaz, M. (2007). Las formas elementales de la pobreza. Madrid: Alianza Editorial.

Peláez-Paz, C. (2014). La quiebra de la clase media española. En Martín Patino, J. M. y Giménez Romero, C. (eds.). Informe España 2014. Una interpretación de su realidad social. Madrid: Fundación Encuentro, pp. 51-100.

Rancière, J. y Panagia, D. (2000). Dissenting words: A conversation with Jacques Rancière. Diacritics, 30 (2), pp. 113-126. https://doi.org/10.1353/dia.2000.0016

Rawls J. (1971). A Theory of Justice. Cambridge, MA: Harvard University Press.

Requena, M. y Stanek, M. (2015). Las clases sociales en España: cambio, composición y consecuencias. En Blanco, A., Chueca, A. y Bombardieri, G. (eds.). Informe España 2015. Una interpretación de su realidad social. Homenaje a José María Martín Patino. Madrid: Fundación Encuentro, pp. 487-517.

Rogers, D. y Bailey, N. (2013). Citizenship and housing: the provision of housing and practices of citizenship within global cities. En Referred Proceedings: 7th Australasian Housing Researchers' Conference. 6th-8th February 2013, 1-14. Fremantle, Western Australia. [En línea]. Disponible en https://bit. ly/2ml2kqi

Sabaté, I. (2014). Del país de los propietarios al país de los sobre-endeudados. Reciprocidad, solidaridad y proyectos de transformación sistémica en tiempos de crisis. Ars y Humanitas, 8 (1), pp. 167-187. https://doi.org/10.4312/ ah.8.1.167-187
Sabaté, I. (2016). The Spanish mortgage crisis and the re-emergence of moral economies in uncertain times. History and Anthropology, 27 (1), pp. 107-120. https://doi.org/10.1080/02757206.201 5.1111882

Sastre, T. y Fernández-Sánchez, J. L. (2011). El ajuste del consumo duradero y no duradero en España durante la crisis económica. Boletín Económico. Banco de España, 1/2011, pp. 89-102.

Schuetz, J., Been, V. y Ellen, I. G. (2008). Neighborhood effects of concentrated mortgage foreclosures. Journal of Housing Economics, 17 (4), pp. 306-319. https://doi.org/10.1016/j. jhe.2008.09.004

Souza Briggs, X. de (1998). Brown kids in white suburbs: Housing mobility and the many faces of social capital. Housing Policy Debate, 9 (1), pp. 177-221. https://doi. org/10.1080/10511482.1998.9521290

Stout, N. (2015, 30 de marzo). Generating home (Theorizing the Contemporary Series). Cultural Anthropology [En línea]. Disponible en: https://culanth. org/fieldsights/generating-home

Suárez, M. (2014). Movimientos sociales y buen vivir: ecuatorianos en la lucha por la vivienda en la plataforma de afectados por la hipoteca (PAH). Revista de Antropología Experimental, 14, pp. 71-89.

Swyngedouw, E. (2005). Governance innovation and the citizen: The Janus face of governance-beyond-the-state. Urban Studies, 42 (11), pp. 1992-2006. https:// doi.org/10.1080/00420980500279869

Swyngedouw, E. (2011). Designing the PostPolitical City and the Insurgent Polis. London: Bedford Press.

Valles, M. S. (1999). Técnicas cualitativas de investigación social. Reflexión metodológica y práctica profesional. Madrid: Síntesis.

Waldron, R. y Redmond, D. (2014). The Extent of the Mortgage Crisis in Ireland and Policy Responses. Housing Studies, 29 (1), pp. 149-165. https://doi.org/10. 1080/02673037.2013.825694

\section{Otros recursos}

Colau, A. y Alemany, A. (2013). 2007-2012: Retrospectiva sobre desahucios y ejecuciones hipotecarias en España, estadisticas oficiales e indicadores. [En línea]. Disponible en https://afectadosporlahipoteca.com//wp-content/ uploads/2013/02/RETROSPECTIVA- 
SOBRE-DESAHUCIOS-Y-EJECUCIONESHIPOTECARIAS-EN-ESPA\%C3\%91A-COLAUALEMANY1.pdf

Comisión Europea. Documento de trabajo de los Servicios de la Comisión. Informe sobre España 2016, con un examen exhaustivo relativo a la prevención y la corrección de los desequilibrios macroeconómicos. [En línea]. Disponible en https://ec.europa.eu/info/sites/info/ files/cr_spain_2016_es.pdf

Emergència habitacional a Catalunya. Impacte de la crisi hipotecària en el dret a la salut $i$ els drets dels infants, 2015. Barcelona: Observatori DESC / Plataforma de Afectados por la Hipoteca.
[En línea]. Disponible en http://observatoridesc.org/sites/default/files/ informe_emergencia_habitacionat_catalunya_2015.pdf

Emergencia habitacional en el Estado español. La crisis de las ejecuciones hipotecarias y los desalojos desde una perspectiva de derechos humanos, 2013. Observatorio DESC / Plataforma de Afectados por la Hipoteca. [En línea]. Disponible en https://afectadosporlahipoteca.com/wp-content/ uploads/2013/12/2013-Informe_habtitatge-17Dic.pdf

Pajares, M. (2010). Inmigración y mercado de trabajo. Informe 2010. Documen- tos del Observatorio Permanente de la Inmigración. Ministerio de Trabajo e Inmigración. [En línea]. Disponible en http://extranjeros.mitramiss.gob.es/ es/ObservatorioPermanentelnmigracion/Publicaciones/fichas/archivos/ Inmigracion__Mercado_de_Trabajo_ OPI25.pdf

Sales i Campos, A. Diagnosis 2013. Las personas sin hogar en la ciudad de Barcelona y la evolución de los recursos de la Red de Atención a Personas sin Hogar. Xarxa d'Atenció a Persones sense Llar, Barcelona. [En línea]. Disponible en http://www.bcn.cat/ barcelonainclusiva/ca/2013/7/xarxa2_diagnosi2013_es.pdf 